\title{
CONTINUOUS HOMOMORPHISMS AND RINGS OF INJECTIVE DIMENSION ONE
}

\author{
SHOU-TE CHANG and I-CHIAU HUANG
}

\begin{abstract}
Let $S$ be an $R$-algebra and a be an ideal of $S$. We define the continuous hom functor from $R$-Mod to $S$-Mod with respect to the a-adic topology on $S$. We show that the continuous hom functor preserves injective modules iff the ideal-adic property and ideal-continuity property are satisfied for $S$ and a. Furthermore, if $S$ is a-finite over $R$, we show that the continuous hom functor also preserves essential extensions. Hence, the continuous hom functor can be used to construct injective modules and injective hulls over $S$ using what we know about $R$. Using the continuous hom functor we can characterize rings of injective dimension one using symmetry for a special class of formal power series subrings. In the Noetherian case, this enables us to construct onedimensional local Gorenstein domains. In the non-Noetherian case, we can apply the continuous hom functor to a generalized form of the $D+M$ construction. We may construct a class of domains of injective dimension one and a series of almost maximal valuation rings of any complete DVR.
\end{abstract}

\section{Introduction}

Throughout this paper, $R, S$ and $T$ are rings, $S$ is an $R$-algebra and $a$ is an ideal of $S$. When $R$ is a domain we always use $Q$ to denote $Q(R)$, the field of fractions of $R$, and $K$ to denote a field containing $Q(R)$. When $X$ is used it always stands for an indeterminate over whatever base ring used. All the rings we consider are commutative with unity 1 and all the modules are unitary. We will also use $S$-Mod and $R$-Mod to denote the category of $S$-modules and the category of $R$-modules respectively.

For any $S$-module $N$ and any $R$-module $M$, the Abelian group $\operatorname{Hom}_{R}(N, M)$ of $R$-linear maps from $N$ to $M$ has a natural $S$-module structure given by $s f=f \circ \mu_{s}$, where $s \in S, f \in \operatorname{Hom}_{R}(N, M)$ and $\mu_{s}$ is the multiplication by $s$. What we are interested in is a subfunctor $\operatorname{Hom}_{R}^{\alpha}(S,-)$ of $\operatorname{Hom}_{R}(S,-)$ from $R$-Mod to $S$-Mod, and we will call this functor the continuous hom functor of $S$ over $R$.

Let $N$ be an $S$-module. Taking $\left\{\alpha+\mathfrak{a}^{n} N\right\}_{n \in \mathrm{Z}_{+}}$as a system of neighborhood of any element $\alpha \in N$, we have a topology on $N$, called the a-adic topology. With respect to the a-adic topology on $S$ and the discrete topology on $M$, the 
$S$-module

$$
\operatorname{Hom}_{R}^{\mathfrak{a}}(S, M)=\left\{f \in \operatorname{Hom}_{R}(S, M): f\left(\mathfrak{a}^{n}\right)=0 \text { for some } n \in \mathrm{Z}_{+}\right\}
$$

is the collection of continuous homomorphisms from $S$ to $M$. The continuous hom functor has been used to construct injective modules when $R \rightarrow S$ is a residually finite local homomorphism of Noetherian local rings. In this particular case, $\operatorname{Hom}_{R}^{\mathfrak{a}}(S,-)$ preserves injective hulls of the residue fields and plays an important role for concrete realization of Grothendieck duality [6], [8]. We aim to find conditions on $S, a$ and $R$ in general for which the continuous hom functor is well-behaving.

In $\S 2$ we investigate when the continuous hom functor preserves injective objects. We may obtain the continuous hom functor as follows. First, apply the functor $\operatorname{Hom}_{R}(S,-): R$-Mod $\rightarrow S$-Mod. It is well-known that this functor preserves injective objects. Next, consider the a-torsion functor defined by $\Gamma_{\mathfrak{a}}(M)=\left\{m \in M: \mathfrak{a}^{n} m=0\right.$ for some $\left.n\right\}$ for any $S$-module $M$. (We remark that the a-torsion functor is used to define local cohomology in the Noetherian case [2]. In the non-Noetherian case, local cohomology is defined by another condition using supports.) Let $\mathscr{A}$ be the full subcategory of $S$-Mod consisting of modules with $\Gamma_{\mathfrak{a}}(M)=M$. We further consider $\Gamma_{\mathfrak{a}}$ as a functor from $S$-Mod to $\mathscr{A}$, which preserves injective objects (cf. [12]). Finally, apply the inclusion functor of $\mathscr{A}$ into $S$-Mod. It is essential to study when this functor preserves injective objects.

In $\S 2$, we also describe two important "topological" conditions for $S$ and $\mathfrak{a}$. The first condition is called the ideal-adic property (Definition 2.1), which is equivalent to a slightly weaker version of the Artin-Rees lemma. It says that the a-adic topology on any $S$-ideal is the same as the inherited subspace topology. The second condition, ideal continuity (see Definition 2.5), basically says that if the restrictions of an $R$-linear map from an ideal $J$ of $S$ to $M$ are continuous on a collection of ideals contained in $J$ which "covers" $J$ as subspaces (note that this is not an open cover) then the $R$-linear map is itself continuous on $J$. Theorem 2.7 tells us that the continuous hom functor preserves injective modules for all $R$ if and only if the inclusion functor of $\mathscr{A}$ into $S$-Mod preserves injective objects if and only if the ideal-adic property and the ideal-continuity property are satisfied for $S$ and $\mathfrak{a}$.

The two topological conditions are automatically satisfied when $S$ is Noetherian. For the rest of $\S 2$, we provide non-Noetherian examples satisfying the two said topological conditions to show that there are plenty of such $S$ and a.

In $\S 3$, we have the second main result, Theorem 3.4, which says when $S$ is a-finite over $R$ (see Definition 3.2) the continuous hom functor of $S$ over $R$ also 
preserves essential extensions. Thus, it gives rise to a method of constructing injective hulls of cyclic modules in Corollary 3.6. With the existence of a trace, Proposition 3.8 pins down our construction to concrete cases. Understanding of injective hulls of cyclic modules is necessary in understanding the structure of injective modules over non-Noetherian rings. Injective hulls are also essential in constructing minimal injective resolutions.

Concrete descriptions of an injective hull for a given module is very subtle, even though they are isomorphic to each other. To appreciate the importance of such concrete descriptions, one may look at concrete aspects of Grothendieck duality, for instance, in the paper [8]. Our philosophy is that important information of a module can be obtained from reading its minimal injective resolution. For an easy example, Kunz's well-known symmetric criterion [10] of the Gorenstein property of numerical semigroup rings $K\left[\left[X^{t_{1}}, \ldots, X^{t_{m}}\right]\right]$ can be obtained from a dualizing complex [7]. The continuous hom functor together with local cohomology play a dominant role in the constructions of injective modules [6], which deals with the Noetherian case. This paper considers the continuous hom functor beyond the Noetherian situation.

In $\S 4$, we apply our results from previous sections to rings of the form

$$
S=R \oplus R_{1} X \oplus \cdots \oplus R_{n-1} X^{n-1} \oplus K[[X]] X^{n} \subset K[[X]] .
$$

The continuous functor makes it possible to construct minimal injective resolutions for this type of rings. In Theorem 4.3, we establish the criterion of symmetry to decide exactly when a ring of this type is of injective dimension one!

Application of Theorem 4.3 in the Noetherian case gives us examples of onedimensional Gorenstein domains. We can recover the Gorenstein numerical semigroup rings described by Kunz [10] using symmetry, or other analytically irreducible local Gorenstein domains obtained by Barucci and Fröberg [1] where symmetry is formulated in terms of dimension formulae. Our approach is to replace the numerical formulae by duality of modules with respect to an injective module and replace the Gorenstein property by finite injective dimension. Thus, our approach naturally extends to the non-Noetherian case. If we apply Theorem 4.3 to rings of the form

$$
S=R \oplus R X \oplus \cdots \oplus R X^{n-1} \oplus Q[[X]] X^{n},
$$

which is a generalization of the $D+M$ construction, we will see that the injectivity of $Q((X)) / S$ is equivalent to the self-duality of $Q$ with respect to $Q / R$ (Definition 4.8). We will show that self-duality of the quotient fields is preserved under such extensions (Proposition 4.12). In particular, we will be able to construct a series of almost maximal valuation rings out of any complete 
DVR's. Furthermore, we provide rings such as

$$
\mathrm{Q}[[t]] \oplus \mathrm{Q}((t)) X^{2} \oplus \mathrm{Q}[[t]] X^{3} \oplus \mathrm{Q}((t))[[X]] X^{4}
$$

and

$$
\mathrm{Q}\left[\left[t^{2}\right]\right] \oplus \mathrm{Q}((t)) X^{2} \oplus\left(\mathrm{Q}\left[\left[t^{2}\right]\right]+\mathrm{Q}\left(\left(t^{2}\right)\right) t\right) X^{3} \oplus \mathrm{Q}((t))[[X]] X^{4}
$$

to give a more general idea how to construct rings of injective dimension 1 (see Examples 4.15 and 4.16). Furthermore, the quotient field of any such ring is self-dual with respect to its first cosyzygy, and the first cosyzygy is an injective hull of the residue field if the domain is quasi-local.

\section{Injective Modules}

In this section we investigate the two topological conditions involving $S$ and $a$ for the continuous hom functor to preserve injective modules.

Let $N$ be an $S$-module. Then a submodule $N^{\prime}$ of $N$ can be endowed with the a-adic topology or the subspace topology inherited from $N$. In general, the a-adic topology is finer than the subspace topology, since $\mathfrak{a}^{n} N^{\prime} \subset \mathfrak{a}^{n} N \cap N^{\prime}$ for any $n \in \mathbf{Z}_{+}$.

Definition 2.1 (Ideal-Adic). The a-adic topology on $S$ is ideal-adic if for any ideal $J$ of $S$ and for any given $n \in \mathrm{Z}_{+}$there exists $m$ such that $\mathfrak{a}^{m} \cap J \subset \mathfrak{a}^{n} J$.

The terminology "ideal-adic" stems from its equivalence to the condition that the subspace topology on any given ideal of $S$ is the same as its a-adic topology.

For the rest of this paper we identify $\operatorname{Hom}_{R}(J, M)$ with $\operatorname{Hom}_{S}\left(J, \operatorname{Hom}_{R}(S\right.$, $M)$ ) via the map which sends an $R$-linear map $f: J \rightarrow M$ to the $S$-linear map $\tilde{f}: J \rightarrow \operatorname{Hom}_{R}(S, M)$ given by $(\tilde{f}(\alpha))(\beta)=f(\alpha \beta)$ for $\alpha \in J$ and $\beta \in S$. Many modules we are interested in will be considered as submodules of $\operatorname{Hom}_{S}\left(J, \operatorname{Hom}_{R}(S, M)\right)$. We will also identify $\operatorname{Hom}_{R}\left(J / \mathfrak{a}^{n} J, M\right)$ with $\{f \in$ $\left.\operatorname{Hom}_{R}(J, M): f\left(\mathfrak{a}^{n} J\right)=0\right\}$.

Definition 2.2 (Continuous Homomorphism). Suppose given an ideal $J$ of $S$ and an $R$-module $M$. Define

$$
\operatorname{Hom}_{R}^{\mathfrak{a}}(J, M):=\bigcup_{n>0} \operatorname{Hom}_{R}\left(J / \mathfrak{a}^{n} J, M\right) \subset \operatorname{Hom}_{R}(J, M) .
$$

The elements in $\operatorname{Hom}_{R}^{\mathfrak{a}}(J, M)$ are called continuous $R$-linear homomorphisms from $J$ to $M$, or we will simply say that $f$ is continuous on $J$. 
For an ideal $J$ of $S$ and an $R$-module $M$, we have

$\operatorname{Hom}_{R}^{\mathfrak{a}}(J, M)=\bigcup_{n>0} \operatorname{Hom}_{S}\left(J, \operatorname{Hom}_{R}\left(S / \mathfrak{a}^{n}, M\right)\right) \subset \operatorname{Hom}_{S}\left(J, \operatorname{Hom}_{R}^{\mathfrak{a}}(S, M)\right)$.

Note that $f \in \operatorname{Hom}_{S}\left(J, \operatorname{Hom}_{R}^{\mathfrak{a}}(S, M)\right)$ if and only if for any $\alpha \in J$, there exists some $n$ such that $f\left(\alpha a^{n}\right)=0$, that is, $f$ is continuous on all principal ideals contained in $J$. Thus, we have the following lemma.

LemMa 2.3. For an ideal $J$ of $S$ and an $R$-module $M$, the following statements are equivalent:

- If an R-linear map $f$ is continuous on all principle ideals contained in $J$ then $f$ is continuous on $J$.

- $\operatorname{Hom}_{R}^{\mathfrak{a}}(J, M)=\operatorname{Hom}_{S}\left(J, \operatorname{Hom}_{R}^{\mathfrak{a}}(S, M)\right)$.

Interestingly, $R$ is not an essential factor in the identity in Lemma 2.3 as we will see in Proposition 2.4. It is elementary to verify that

$$
\operatorname{Hom}_{R}^{\mathfrak{a}}(J, M)=\operatorname{Hom}_{S}^{\mathfrak{a}}\left(J, \operatorname{Hom}_{R}(S, M)\right)
$$

for any ideal $J$ of $S$ and any $R$-module $M$.

Proposition 2.4. The following statements are equivalent for all ideals $J$ of $S$ :

(a) $\operatorname{Hom}_{\mathrm{Z}}^{\mathfrak{a}}(J, M)=\operatorname{Hom}_{S}\left(J, \operatorname{Hom}_{\mathrm{Z}}^{\mathfrak{a}}(S, M)\right)$ for any Z-module $M$;

(b) $\operatorname{Hom}_{R}^{\mathfrak{a}}(J, M)=\operatorname{Hom}_{S}\left(J, \operatorname{Hom}_{R}^{\mathfrak{a}}(S, M)\right)$ for any ring $R$ such that $S$ is an $R$-algebra and any $R$-module $M$;

(c) $\operatorname{Hom}_{R}^{\mathfrak{a}}(J, M)=\operatorname{Hom}_{S}\left(J, \operatorname{Hom}_{R}^{\mathfrak{a}}(S, M)\right)$ for some ring $R$ such that $S$ is an $R$-algebra and any $R$-module $M$;

(d) $\operatorname{Hom}_{S}^{a}(J, M)=\operatorname{Hom}_{S}\left(J, \operatorname{Hom}_{S}^{a}(S, M)\right)$ for any $S$-module $M$.

Proof. (a) $\Rightarrow$ (b) $\Rightarrow$ (c) $\Rightarrow$ (d) is trivial. We now prove (d) $\Rightarrow$ (a). Let $M$ be a Z-module and let $\mathfrak{M}=\operatorname{Hom}_{\mathrm{Z}}(S, M)$. Using identity (1), we have $\operatorname{Hom}_{\mathrm{Z}}^{\mathfrak{a}}(S, M)$ $=\operatorname{Hom}_{S}^{\mathfrak{a}}(S, \mathfrak{M})$. By repeated use of identity (1), we have $\operatorname{Hom}_{\mathrm{Z}}^{\mathfrak{\alpha}}(J, M)=$ $\operatorname{Hom}_{S}^{\mathfrak{a}}(J, \mathfrak{M})=\operatorname{Hom}_{S}\left(J, \operatorname{Hom}_{S}^{\mathfrak{a}}(S, \mathfrak{M})\right)=\operatorname{Hom}_{S}\left(J, \operatorname{Hom}_{\mathrm{Z}}^{\mathfrak{a}}(S, M)\right)$.

Definition 2.5 (Ideal-Continuity). The a-adic topology on $S$ admits idealcontinuity if one of the equivalent statements in Proposition 2.4 holds for all ideals $J$ of $S$.

Remember that the category $\mathscr{A}$ is the full subcategory of $S$-Mod consisting of modules with $\Gamma_{\mathfrak{a}}(M)=M$. It is easy to see that $\mathscr{A}$ is an abelian category.

Lemma 2.6. The functor $\Gamma_{\mathfrak{a}}: S$-Mod $\rightarrow \mathscr{A}$ preserves injective objects (cf. [12, Ex. 4.6.3]). 
Proof. Let $E$ be an injective $S$-module and let $N \subset M$ be objects of $\mathscr{A}$. For any $S$-linear map $f: N \rightarrow \Gamma_{\mathfrak{a}}(E)$, the map $N \stackrel{f}{\rightarrow} \Gamma_{\mathfrak{a}}(E) \hookrightarrow E$ can be extended to an $S$-linear map $g: M \rightarrow E$. Obviously, $g(M) \subset \Gamma_{\mathfrak{a}}(E)$. Hence, $g$ factors through $\Gamma_{\mathfrak{a}}(E)$ and gives rise to an extension of $f$ to $M$.

Next is the main result of this section.

THEOREM 2.7. The following statements are equivalent.

(a) The inclusion functor of $\mathscr{A}$ into $S$-Mod preserves injective objects.

(b) The functor $\operatorname{Hom}_{R}^{\mathfrak{a}}(S,-)$ preserves injective modules for all rings $R$ such that $S$ is an $R$-algebra.

(c) The functor $\operatorname{Hom}_{S}^{\mathfrak{a}}(S,-)$ preserves injective modules.

(d) The a-adic topology on $S$ is ideal-adic and admits ideal-continuity.

Proof. By the discussion on $\Gamma_{\mathfrak{a}}$ and $\mathscr{A},(\mathrm{a}) \Rightarrow(\mathrm{b})$ is clear. The implication (b) $\Rightarrow$ (c) is trivial.

(c) $\Rightarrow$ (a): Let $E$ be an injective object in $\mathscr{A}$. Find an injective hull $E^{\prime}$ of $E$ in $S$-Mod. Then $\Gamma_{\mathfrak{a}}\left(E^{\prime}\right)=E \oplus E^{\prime \prime}$ in $\mathscr{A}$. Being a submodule of an injective hull of $E, E \oplus E^{\prime \prime}$ is an essential extension of $E$. Thus $E^{\prime \prime}=0$. By (c), $E=\Gamma_{\mathfrak{a}}\left(E^{\prime}\right) \cong \operatorname{Hom}_{S}^{\mathfrak{a}}\left(S, E^{\prime}\right)$ is injective in $S$-Mod.

(a) and (c) $\Rightarrow(\mathrm{d})$ : Let $J$ be an ideal of $S$. Find an injective $S$-module $E$ containing $J / \mathfrak{a}^{n} J$. Then $E^{\prime}=\Gamma_{\mathfrak{a}}(E)$ is an injective object containing $J / \mathfrak{a}^{n} J$ in $\mathscr{A}$ by Lemma 2.6. By (a), $E^{\prime}$ is also injective in $S$-Mod. The $S$-linear map $J \rightarrow J / \mathfrak{a}^{n} J \stackrel{\iota}{\hookrightarrow} E^{\prime}$ can be extended to an $S$-linear map $S \stackrel{g}{\rightarrow} E^{\prime}$. Since $g(1)$ is killed by $\mathfrak{a}^{m}$ for some $m$, we have $g\left(\mathfrak{a}^{m} S\right)=0$, and so $g$ factors as $S \rightarrow S / \mathfrak{a}^{m} \stackrel{\tilde{g}}{\rightarrow} E^{\prime}$. Now let $\alpha \in J \cap \mathfrak{a}^{m}$. Then $\iota\left(\alpha+\mathfrak{a}^{n} J\right)=\widetilde{g}\left(\alpha+\mathfrak{a}^{m}\right)=0$. This implies that $\alpha \in \mathfrak{a}^{n} J$, that is, $\mathfrak{a}^{m} \cap J \subset \mathfrak{a}^{n} J$. Thus, the a-topology on $S$ is ideal-adic.

To show the a-adic topology on $S$ admits ideal-continuity, take an $S$-linear map $f: J \rightarrow \operatorname{Hom}_{S}^{\mathfrak{a}}(S, M)$. We embed $M$ into an injective $S$-module $E$. By the injectivity of $\operatorname{Hom}_{S}^{\mathfrak{a}}(S, E)$, the map $J \stackrel{f}{\rightarrow} \operatorname{Hom}_{S}^{\mathfrak{a}}(S, M) \rightarrow \operatorname{Hom}_{S}^{\mathfrak{a}}(S, E)$ extends to some $g: S \rightarrow \operatorname{Hom}_{S}^{\mathfrak{a}}(S, E)$. Since $g(1)$ is killed by $\mathfrak{a}^{n}$ for some $n$, we have $g\left(\mathfrak{a}^{n}\right)=0$. Thus, $f\left(\mathfrak{a}^{n} J\right)=g\left(\mathfrak{a}^{n} J\right)=0$ and $f \in \operatorname{Hom}_{S}^{\mathfrak{a}}\left(J, \operatorname{Hom}_{R}(S\right.$, $M))=\operatorname{Hom}_{S}^{\mathfrak{a}}(J, M)$. We have proved that $\operatorname{Hom}_{S}^{\mathfrak{a}}(J, M)=\operatorname{Hom}_{S}(J$, $\left.\operatorname{Hom}_{S}^{\mathfrak{a}}(S, M)\right)$.

(d) $\Rightarrow$ (a): Let $E$ be an injective object in $\mathscr{A}$. Note that in this case $E \cong$ $\operatorname{Hom}_{S}(S, E)=\operatorname{Hom}_{S}^{\mathfrak{a}}(S, E)$. If $J$ be an ideal of $S$ and $f \in \operatorname{Hom}_{S}(J, E)$, then the ideal-continuity property guarantees that $f \in \operatorname{Hom}_{S}\left(J, \operatorname{Hom}_{S}^{\mathfrak{a}}(S, E)\right)=$ $\operatorname{Hom}_{S}^{a}(J, E)$, and so we have that $f: J \rightarrow E$ factors as $f: J \rightarrow J / \mathfrak{a}^{n} J \rightarrow E$ 
for some $n$. Find $m$ such that $\mathfrak{a}^{m} \cap J \subset \mathfrak{a}^{n} J$. The map $f$ factors as

$$
J \rightarrow J /\left(\mathfrak{a}^{m} \cap J\right) \rightarrow J / \mathfrak{a}^{n} J \rightarrow E .
$$

Since $J /\left(\mathfrak{a}^{m} \cap J\right)$ is a submodule of $S / \mathfrak{a}^{m}$ in $\mathscr{A}, J /\left(\mathfrak{a}^{m} \cap J\right) \rightarrow J / \mathfrak{a}^{n} J \rightarrow E$ can be extended to $g: S /\left(\mathfrak{a}^{m} \cap J\right) \rightarrow E$. The composite $S \rightarrow S /\left(\mathfrak{a}^{m} \cap J\right) \stackrel{g}{\rightarrow} E$ is an extension of $f$ in $S$-Mod. Thus $E$ is injective in $S$-Mod.

It is quite obvious when $S$ is Noetherian, the a-adic topology on $S$ is idealadic and admits ideal-continuity for any ideal $\mathfrak{a}$ of $S$. For non-Noetherian examples, we introduce the following notion.

Definition 2.8. Let $S$ be a subring of $T$. If $\mathfrak{a}$ is both an ideal of $S$ and an ideal of $T$, we say that $\mathfrak{a}$ is an ideal of $S$ along $T$.

It is clear that $\mathfrak{a}=\mathfrak{a} T$ if $\mathfrak{a}$ is an ideal of $S$ along $T$. Note that in this case $J \mathfrak{a}$ for any ideal $J$ of $S$ and any power of a are also ideals of $S$ along $T$.

Proposition 2.9. Let $S$ be a subring of a Noetherian ring $T$, and let a be an ideal of $S$ along $T$. Then the a-adic topology on $S$ is ideal-adic and admits ideal-continuity.

Proof. Suppose given any ideal $J$ of $S$. By the Artin-Rees lemma for $T$, for any $n$ there exists $m$ such that $\mathfrak{a}^{m} \cap J \subset \mathfrak{a}^{m} T \cap J T \subset \mathfrak{a}^{n} J T=\mathfrak{a}^{n} J$ since $\mathfrak{a}=\mathfrak{a} T$. Hence the a-topology on $S$ is ideal-adic.

If $f: J \rightarrow M$ is an $R$-linear homomorphism which is continuous on $\alpha S$ for every $\alpha \in J$, then $\left.f\right|_{\alpha J}$ is also continuous on $\beta S$ for every $\beta \in \mathfrak{a} J$. Since $a J=a J T$ is also an ideal of $T$, from the Noetherian case we have that $\left.f\right|_{a J T}$ is continuous with respect to the a-adic topology on $T$, that is, $f\left(\mathfrak{a}^{n}(\mathfrak{a} J)\right)=f\left(\mathfrak{a}^{n}(\mathfrak{a} J T)\right)=0$ for some $n$. Hence $f$ is continuous on $J$ with respect to a-adic topology on $S$.

Let $S$ be a subring of $K[[X]]$. The conductor a of $S$ along $K[[X]]$ is the largest $K[[X]]$-ideal contained in $S$, that is,

$$
\mathfrak{a}=\{\varphi \in S: \varphi K[[X]] \subset S\} .
$$

The conductor $\mathfrak{a}$ is proper (that is, $\mathfrak{a} \neq S$ or equivalently $\mathfrak{a} \neq K[[X]]$ ) if and only $S$ is a proper subring of $K[[X]]$. If $\mathfrak{a}$ is non-zero, it is of the form $K[[X]] X^{n}$ for some $n \geq 0$. By Proposition 2.9, the a-adic topology on $S$ is ideal-adic and admits ideal-continuity.

The class of rings ideal-adic and admitting ideal-continuity is not restricted to those in Proposition 2.9. For example, let $S$ be the localization of the ring $K\left[X^{r}: 0<r \in \mathrm{R}\right]$ at the maximal ideal $\left(X^{r}: 0<r \in \mathrm{R}\right)$. We denote $X^{0}:=1$. Every nonzero element of $S$ can be written as $X^{r} \phi$, where $\phi$ is a unit 
of $S$. Thus an ideal $I$ of $S$ is generated by those $X^{r}$ with $X^{r} \in I$. For a real number $c \geq 0$, we denote by $I_{c}$ the ideal of $S$ generated by those $X^{r} \in S$ with $r \geq c$; we denote by $I_{c}^{+}$the ideal of $S$ generated by those $X^{r} \in S$ with $r>c$. A non-zero ideal of $S$ is either of the form $I_{c}$ or of the form $I_{c}^{+}$. For $c \in \mathrm{R}$, the ideal $I_{c}^{+}$is not finitely generated while $I_{c}$ is principal. It is easy to see that

(a) $I_{c}^{+} \subsetneq I_{c} \subsetneq I_{d}^{+} \subsetneq I_{d}$ for $d<c$;

(b) $I_{c} I_{d}=I_{c+d}$ and $I_{c} I_{d}^{+}=I_{c}^{+} I_{d}^{+}=I_{c+d}^{+}$.

Proposition 2.10. Let $S$ be as above and $\mathfrak{a}=I_{c}$ or $I_{c}^{+}$where $c>0$. Then the a-adic topology of $S$ is ideal-adic and admits ideal-continuity. If $\mathfrak{a}=I_{c}^{+}$, then a is not an ideal of any Noetherian ring containing $S$.

Proof. Take any nonzero proper ideal $J=I_{d}$ or $I_{d}^{+}$. For any $n$, choose $m$ such that $m c>n c+d$. Then $\mathfrak{a}^{m} \cap J \subset I_{m c} \subset I_{n c+d}^{+} \subset \mathfrak{a}^{n} J$. Hence the a-adic topology of $S$ is ideal-adic. Now let $M$ be any $S$-module and let $f \in \operatorname{Hom}_{S}(J, M)$ be such that $f$ is continuous on $S \alpha$ for all $\alpha \in J$. In particular, suppose $X^{r} \in J$ and $f\left(a^{n} X^{r}\right)=0$ for some $n$. Find $m$ such that $m c+d>n c+r$. Then $\mathfrak{a}^{m} J \subset I_{m c+d} \subset I_{n c+r}^{+} \subset \mathfrak{a}^{n} X^{r}$. Hence $f\left(\mathfrak{a}^{m} J\right)=0$, that is, $f \in \operatorname{Hom}_{S}^{\mathfrak{a}}(J, M)$. Therefore, the $\mathfrak{a}$-adic topology of $S$ admits idealcontinuity.

Assume that $\mathfrak{a}=I_{c}^{+}$is an ideal of a Noetherian ring $T$ containing $S$. We may choose $X^{r_{1}}, \ldots, X^{r_{s}}$ which generate $\mathfrak{a}$ as an ideal of $T$. With $r=\min \left\{r_{i}\right.$ : $i=1, \ldots, s\}$, we have $\mathfrak{a}=T X^{r}$. Since $r>c$, there exists a positive integer $n$ such that $r-(1 / n)>c$. Let $\alpha$ be an element in $T$ such that $\alpha X^{r}=X^{r-(1 / n)}$. Choose a positive integer $m>c$. Then $\alpha^{m n} X^{m} \in T \mathfrak{a}=\mathfrak{a} \subset S$. On the other hand, $\alpha^{m n} X^{m} X^{r m n}=\left(\alpha X^{r}\right)^{m n} X^{m}=X^{r m n}$. Since $S$ is a domain, we get the contradiction that $1=\alpha^{m n} X^{m} \in \mathfrak{a}$.

\section{Injective Hulls}

To construct injective hulls, we need to study essential extensions of modules under the continuous hom functor. It turns out that certain topological finiteness is sufficient for the continuous hom functor to preserve essential extensions.

Definition 3.1. Let $\mathfrak{b}$ be an ideal of $S$. We say that $\mathfrak{a}$ and $\mathfrak{b}$ are adically equivalent if and only if a power of $\mathfrak{b}$ is contained in $\mathfrak{a}$ and a power of $\mathfrak{a}$ is contained in $\mathfrak{b}$.

Remark. When two ideals $\mathfrak{a}$ and $\mathfrak{b}$ of $S$ are adically equivalent, the $\mathfrak{b}$-adic topology is the same as the $a$-adic topology. Any property satisfied by the $\mathfrak{a}$-adic topology is also satisfied by the $\mathfrak{b}$-adic topology. In particular, $\operatorname{Hom}_{R}^{\mathfrak{a}}(S, M)=$ $\operatorname{Hom}_{R}^{\mathfrak{b}}(S, M)$ for any $R$-module $M$. 
Theorem 3.4 is the main result for this section. Note that neither the idealadic nor the ideal-continuity property is required.

Definition 3.2 (Adic Finite). The a-adic topology on $S$ is finite over $R$, or $S$ is $\mathfrak{a}$-finite over $R$, if $S / \mathfrak{b}$ is a finite $R$-module for some ideal $\mathfrak{b}$ of $S$ which is adically equivalent to $\mathfrak{a}$.

Lemma 3.3. Let $\mathfrak{b}$ be any ideal of $S$ which is adically equivalent to $\mathfrak{a}$. Then $\operatorname{Hom}_{R}(S / \mathfrak{b}, M) \subseteq \operatorname{Hom}_{R}^{\mathfrak{a}}(S, M)$ is essential for any $R$-module $M$.

Proof. The inclusion is obvious. Suppose given any non-zero continuous homomorphism $f \in \operatorname{Hom}_{R}^{\mathfrak{a}}(S, M)$. Then $f$ must annihilate some power of $\mathfrak{b}$. Find the smallest $n$ such that $f\left(\mathfrak{b}^{n}\right)=0$, and find $a \in \mathfrak{b}^{n-1} \backslash \mathfrak{b}^{n}$ such that $f(a) \neq 0$. Then $a f$ is a non-zero element in $\operatorname{Hom}_{R}(S / \mathfrak{b}, M)$.

THEOREM 3.4. Let $S$ be an R-algebra. If $S$ is a-finite over $R$, the functor $\operatorname{Hom}_{R}^{\mathfrak{a}}(S,-)$ preserves essential extensions.

Proof. Let $\mathfrak{b}$ be an ideal of $S$ which is adically equivalent to $a$ such that $S / \mathfrak{b}$ is a finite $R$-module. Let $M \subset E$ be an essential extension of $R$-modules and let $e_{1}, \ldots, e_{n}$ be generators of $S / \mathfrak{b}$ as an $R$-module. Given a non-zero $f \in \operatorname{Hom}_{R}(S / \mathfrak{b}, E)$, we choose

$$
b_{1}= \begin{cases}1, & \text { if } f\left(e_{1}\right)=0 ; \\ \text { an element of } R \text { such } & \text { if } f\left(e_{1}\right) \neq 0 . \\ \text { that } b_{1} f\left(e_{1}\right) \in M \backslash\{0\}, & \end{cases}
$$

For $1 \leq i \leq n-1$, we choose $b_{i+1} \in R$ inductively from $b_{1}, \ldots, b_{i}$. Let

$$
b_{i+1}= \begin{cases}1, & \text { if } b_{1} b_{2} \ldots b_{i} f\left(e_{i+1}\right)=0 ; \\ \text { an element of } R \text { such that } & \text { otherwise. } \\ b_{1} b_{2} \ldots b_{i+1} f\left(e_{i+1}\right) \in M \backslash\{0\}, & \end{cases}
$$

We obtain an element $b_{1} b_{2} \ldots b_{n} \in R$ such that $b_{1} b_{2} \ldots b_{n} f \in \operatorname{Hom}_{R}(S / \mathfrak{b}$, $M)$. We see by induction on $i$ that $b_{1} b_{2} \ldots b_{i} f$ is not zero, since, in each step either $b_{1} \ldots b_{i} b_{i+1} f\left(e_{j}\right)=b_{1} \ldots b_{i} f\left(e_{j}\right) \neq 0$ for some $j \neq i+1$ or $b_{1} \ldots b_{i+1} f\left(e_{i+1}\right) \neq 0$. Hence $\operatorname{Hom}_{R}(S / \mathfrak{b}, E)$ is essential over $\operatorname{Hom}_{R}(S / \mathfrak{b}$, $M)$. By Lemma 3.3, the extension $\operatorname{Hom}_{R}^{\mathfrak{a}}(S, E) \supset \operatorname{Hom}_{R}(S / \mathfrak{b}, E)$ is essential. We conclude that $\operatorname{Hom}_{R}^{\mathfrak{a}}(S, E)$ is essential over $\operatorname{Hom}_{R}(S / \mathfrak{b}, M)$, and hence also essential over $\operatorname{Hom}_{R}^{\mathfrak{a}}(S, M)$.

Combining results from $\S 2$ and this section, we have the following result.

Corollary 3.5. Assume that the a-adic topology on $S$ is ideal-adic, admits ideal-continuity and is finite over $R$. If $E$ is an injective hull of $M$ over $R$, then 
$\operatorname{Hom}_{R}^{\mathfrak{a}}(S, E)$ is an injective hull of $\operatorname{Hom}_{R}^{\mathfrak{a}}(S / \mathfrak{b}, M)$ over $S$ where $\mathfrak{b}$ is an ideal adically equivalent to a.

Next, we concentrate on constructing injective hulls of cyclic modules over $S$. This is particularly important in the non-Noetherian case since in that case it is not enough to only study injective hulls of residue fields of local rings.

Corollary 3.6. Assume that the a-adic topology on $S$ is ideal-adic, admits ideal-continuity and is finite over $R$. Let $I$ be an ideal of $R$ and $\sigma \in \operatorname{Hom}_{R}^{\mathfrak{a}}(S, R / I)$. If $\operatorname{Hom}_{R}^{\mathfrak{a}}(S, R / I)$ is essential over $S \sigma$, then $\operatorname{Hom}_{R}^{\mathfrak{a}}(S, E)$ is an injective hull of $S /$ Ann $\sigma$ for any injective hull $E$ of $R / I$.

The notion of trace occurs in constructions of injective hulls.

Definition 3.7 (Trace). Let $A$ be a commutative ring and $B$ be an $A$ algebra. A torsion free element $\sigma$ in $\operatorname{Hom}_{A}(B, A)$ is a trace of $B$ if $\operatorname{Hom}_{A}(B, A)$ $=B \sigma$.

The reader is referred to [9, Appendix F] for discussions on the connection between the "classical traces" and "traces" in the sense of Definition 3.7.

Proposition 3.8. Assume that the a-adic topology on $S$ is ideal-adic, admits ideal-continuity and is finite over $R$. Let $I$ be an ideal of $R$ such that $S /(I S+\mathfrak{a})$ has a trace as an $R / I$-algebra. Then $\operatorname{Hom}_{R}^{\mathfrak{a}}(S, E)$ is an injective hull of $S /(I S+a)$ over $S$ for any injective hull $E$ of $R / I$ over $R$.

Proof. By Lemma 3.3, $\operatorname{Hom}_{R}^{\mathfrak{a}}(S, R / I)$ is essential over $\operatorname{Hom}_{R}(S / \mathfrak{a}, R / I)$. Since $I(S / \mathfrak{a})$ is in the kernel of any $R$-linear map $S / \mathfrak{a} \rightarrow R / I$, the canonical map

$$
\operatorname{Hom}_{R}(S /(I S+\mathfrak{a}), R / I) \rightarrow \operatorname{Hom}_{R}(S / \mathfrak{a}, R / I)
$$

is an isomorphism. A map $S /(I S+\mathfrak{a}) \rightarrow R / I$ is $R$-linear if and only if it is $R / I$-linear, i.e., $\operatorname{Hom}_{R / I}(S /(I S+\mathfrak{a}), R / I)=\operatorname{Hom}_{R}(S /(I S+\mathfrak{a}), R / I)$. A trace $\sigma$ of $S /(I S+\mathfrak{a})$ gives a required isomorphism $\operatorname{Hom}_{R}(S / \mathfrak{a}, R / I) \simeq$ $S \sigma \simeq S /(I S+\mathfrak{a})$. Now the result follows from Corollary 3.6.

A local homomorphism is residually finite if the induced field extension on the residue fields is finite. If $R \rightarrow S$ is residually finite, a trace of the residue field of $S$ over the residue field of $R$ always exists.

Corollary 3.9 ([6, Proposition 3.4]). If $(R, \mathfrak{m}) \rightarrow(S, \mathfrak{n})$ is a residually finite local homomorphism of Noetherian local rings, then $\operatorname{Hom}_{R}^{\mathfrak{n}}(S, E)$ is an injective hull of $S / \mathfrak{n}$ over $S$, where $E$ is an injective hull of $R / \mathfrak{m}$ over $R$. 


\section{Symmetry and Self-duality}

In this section we study the following class of subrings of $K[[X]]$ (2)

$$
\begin{cases}S=R_{0} \oplus R_{1} X \oplus \cdots \oplus R_{n-1} X^{n-1} \oplus K[[X]] X^{n}, & \text { where } R_{n-1} \subsetneq K ; \\ R=R_{0} ; \mathfrak{a}=K[[X]] X^{n} ; & n \geq 1 .\end{cases}
$$

Throughout this section, we will use the convention that $R_{i}=K$ for $i \geq n$ and $R_{i}=0$ for $i<0$. Note that $Q(S)=K((X))$ and $\mathfrak{a}$ is the conductor of $S$. Hence by Proposition 2.9, the a-adic topology on $S$ is ideal-adic and admits ideal-continuity. Since the ideal $\mathfrak{b}=S \cap X K[[X]]$ is adically equivalent to a, $S$ is also a-finite over $R$. We want to study when such a ring is of injective dimension one, that is, when $K((X)) / S$ is injective.

Let $M$ be an $R$-module. Since any function in $\operatorname{Hom}_{R}^{\mathfrak{a}}(S, M)$ vanishes on a sufficiently high power of $\mathfrak{a}, \operatorname{Hom}_{R}^{\mathfrak{a}}(S, M)$ can be identified with $\bigoplus_{i \geq 0} \operatorname{Hom}_{R}\left(R_{i} X^{i}, M\right)$. By the $S$-module structure of $\operatorname{Hom}_{R}^{\mathfrak{a}}(S, M)$, it is natural to denote an element $f$ in $\operatorname{Hom}_{R}\left(R_{i} X^{i}, M\right)$ as $f_{i} X^{-i}$ where $f_{i} \in$ $\operatorname{Hom}_{R}\left(R_{i}, M\right)$ is given by $f_{i}(a)=f\left(a X^{i}\right)$ for $a \in R_{i}$. Hence we will write

$$
\operatorname{Hom}_{R}^{\mathfrak{a}}(S, M)=\bigoplus_{i \geq 0} \operatorname{Hom}_{R}\left(R_{i}, M\right) X^{-i} .
$$

If $j \leq i$ and $a_{j} \in R_{j}$ then $\left(a_{j} X^{j}\right)\left(f_{i} X^{-i}\right)=\left(a_{j} f_{i}\right) X^{j-i}$ where $a_{j} f_{i} \in$ $\operatorname{Hom}_{R}\left(R_{i-j}, M\right)$. If $j>i$, then $\left(a_{j} X^{j}\right)\left(f_{i} X^{-i}\right)=0$.

For the rest of this section we will use $M^{\vee}$ to denote $\operatorname{Hom}_{R}\left(M, K / R_{n-1}\right)$ for any $R$-module $M$ unless otherwise noted. There is a well-defined canonical map $K / R_{n-1-i} \rightarrow R_{i}^{\vee}$ which sends $\bar{a} \in K / R_{n-1-i}$ to the map $b \mapsto \overline{a b}$.

Definition 4.1. We say $S$ as in (2) is symmetric if the canonical maps $K / R_{n-1-i} \rightarrow R_{i}^{\vee}$ are isomorphisms for all $i$.

Remark. For $i<0$, both $K / R_{n-1-i}$ and $R_{i}$ are 0 and naturally $K / R_{n-1-i}$ $\rightarrow R_{i}^{\vee}$ is an isomorphism. For $i \geq n$, both $K / R_{n-1-i}$ and $R_{i}$ are $K$. The canonical map $K \rightarrow K^{\vee}$ is always a monomorphism.

Lemma 4.2. Let $S$ be a subring of $T[[X]]$ and $R=S /(S \cap X T[[X]])$. Let $\mathfrak{M}$ be an $S$-module and $M$ be the submodule of elements in $M i$ which are killed by $S \cap X T[[X]]$. If $M$ is injective over $S$ then $M$ is injective over $R$.

Proof. Since $M \cong \operatorname{Hom}_{S}(R, \mathfrak{M})$, it is injective over $R$ if $\mathfrak{M}$ is injective over $S$.

THEOREM 4.3 (Criterion of symmetry). Let $S, R$ and $K$ be as given in (2). Then $S$ is of injective dimension 1 if and only if $K / R_{n-1}$ is an injective $R$ module and $S$ is symmetric. 
Proof. Let $a$ be as given in (2). The canonical maps $K / R_{n-1-i} \rightarrow R_{i}^{\vee}$ induce a canonical homogeneous $S$-linear map

(3)

$$
\Psi: \frac{K((X))}{S}=\bigoplus_{i \geq 0}\left(\frac{K}{R_{n-1-i}}\right) X^{n-1-i} \longrightarrow \operatorname{Hom}_{R}^{\mathfrak{a}}\left(S, \frac{K}{R_{n-1}}\right) \simeq \bigoplus_{i \geq 0} R_{i}^{\vee} X^{-i}
$$

with a negative degree shift by $n-1$.

When $S$ is symmetric, the map $\Psi$ in (3) is an isomorphism. Thus $K((X)) / S$ is isomorphic to $\operatorname{Hom}_{R}^{\mathfrak{a}}\left(S, K / R_{n-1}\right)$ which is injective by Theorem 2.7 when $K / R_{n-1}$ is injective.

Now assume $S$ is of injective dimension 1, that is, we assume $K((X)) / S$ is injective. Since $K / R_{n-1}$ is isomorphic to the submodule $K / R_{n-1} X^{n-1}$ inside $K((X)) / S$ which is killed by $S \cap X K[[X]]$, it is an injective $R$-module by Lemma 4.2. To show that $S$ is symmetric, it suffices to show that $\Psi$ is an isomorphism. As $S$-modules, $\operatorname{Hom}_{R}^{\mathfrak{a}}\left(S, K / R_{n-1}\right)$ is an injective hull of $R_{0}^{\vee} \cong K / R_{n-1}$ using Corollary 3.5 and $\mathfrak{b}=S \cap X K[[X]]$. Being injective, $K((X)) / S$ contains a submodule $E$ containing $\left(K / R_{n-1}\right) X^{n-1}$ and isomorphic to $\operatorname{Hom}_{R}^{\mathfrak{a}}\left(S, K / R_{n-1}\right)$. The restriction $\left.\Psi\right|_{E}$ of the canonical map (3) is one-to-one, since $E$ is essential over $\left(K / R_{n-1}\right) X^{n-1}$ and the restriction of $\Psi$ to $\left(K / R_{n-1}\right) X^{n-1}$ is one-to-one. Furthermore, the restriction $\left.\Psi\right|_{E}$ is onto, since $\operatorname{Hom}_{R}^{\mathfrak{a}}\left(S, K / R_{n-1}\right)$ is an injective hull of $R_{0}^{\vee}=\Psi\left(\left(K / R_{n-1}\right) X^{n-1}\right)$ and $\Psi(E)$ is an injective module. Thus $\Psi$ is onto and we have a decomposition $K((X)) / S=E \oplus \operatorname{ker} \Psi$ with $\operatorname{ker} \Psi$ being an injective module. We claim that $\operatorname{ker} \Psi=0$. Since for $i \geq n$ and $i<0$, the map $K / R_{n-1-i} \rightarrow R_{i}^{\vee}$ is an injection, $\operatorname{ker} \Psi$ is a submodule of $\oplus_{0 \leq i \leq n-2}\left(K / R_{i}\right) X^{i}$. This implies that $X^{n-1} \operatorname{ker} \Psi=0$. But $\operatorname{ker} \Psi$ is divisible, which means that every element of ker $\Psi$ can be written as $X^{n-1} f$ for some $f \in \operatorname{ker} \Psi$. Therefore $\operatorname{ker} \Psi=0$.

Corollary 4.4. Let $S, R, K$ and a be as given in (2). If $S$ is of injective dimension 1, then the first cosyzygy $K((X)) / S$ of $S$ is isomorphic to $\operatorname{Hom}_{R}^{\mathfrak{a}}(S, K / R)$.

Before applying the criterion of symmetry, we first establish when $S$ as given in (2) would be Noetherian.

Proposition 4.5. Let $S$ be a subring of $T[[X]]$ such that $S \cong R \oplus(S \cap$ $X T[[X]])$ and $X^{n} T[[X]] \subset S$ for some $n$. The following two conditions are equivalent:

(a) $S$ is a Noetherian ring;

(b) $R$ is a Noetherian ring and $T$ is a finitely generated $R$-module.

Proof. First assume (a). The ring $R$ as a quotient of $S$ is Noetherian as well. 
Choose finitely many power series $a_{n j} X^{n}+a_{(n+1) j} X^{n+1}+\cdots$ generating the ideal $X^{n} T[[X]]$ of $S$. Then $T$ is finitely generated by $a_{n j}$ as an $R$-module.

Now we assume (b). Suppose given a non-zero ideal $J$ of $S$. Let $I_{i}$ be the $R$-module consisting of elements $b$ with certain $b X^{i}+b_{i+1} X^{i+1}+\cdots \in J$. Since $X^{n} \in S$, we have that $I_{i} \subseteq I_{i+n} \subseteq I_{i+2 n} \subseteq \cdots$ is an ascending chain of $R$-submodules of $T$ for each $i$. Find $m$ large enough so that $I_{i+k n}=I_{i+(m-1) n}$ for all $0 \leq i \leq n-1$ and $k \geq m$. Being a submodule of $T, I_{i}$ is finitely generated for each $i$, and so we can find finitely many power series $f_{i j}=$ $b_{i j} X^{i}+b_{(i+1) j} X^{i+1}+\cdots \in J$ so that $b_{i j}$ generate $I_{i}$ over $R$. Then $J$ is finitely generated by $f_{i j}$ where $i<m n$. Indeed, any element $g \in J$ may be written as

$$
g=\sum_{i<(m-1) n} a_{i j} f_{i j}+\sum_{(m-1) n \leq i<m n} g_{i j} f_{i j},
$$

where $a_{i j} \in R$ and $g_{i j} \in R\left[\left[X^{n}\right]\right] \subset S$.

Remark. (1) To show "(b) $\Rightarrow$ (a)", it is sufficient to assume $R\left[\left[X^{n}\right]\right] \subset S$ for some $n$, instead of assuming $X^{n} T[[X]] \subset S$.

(2) This proposition for the case when $S=R \oplus X T[[X]]$, is the main result in [5]. It also generalizes the Noetherian criterion for generalized semigroup rings [1, Proposition 7].

By Proposition 4.5, the ring $S$ in (2) is Noetherian if and only if it is of the form in the next corollary. We recover a result by Barucci and Fröberg [1, Theorem 15], which also follows from results by Campillo, Delgado and Kiyek [3].

Corollary 4.6. Let

$$
S=\kappa \oplus V_{1} X \oplus \cdots \oplus V_{n-1} X^{n-1} \oplus X^{n} K[[X]]
$$

be a ring where $\kappa \subset K$ is a finite field extension, $V_{i}$ is a subspace of $K$ for $1 \leq i \leq n-1$ and $V_{n-1} \neq K$. Then $S$ is Gorenstein if and only if $\operatorname{dim}_{\kappa} V_{i}+\operatorname{dim}_{\kappa} V_{n-1-i}=\operatorname{dim}_{\kappa} K$.

Proof. Write $\operatorname{dim}$ for $\operatorname{dim}_{\kappa}$ and let $d=\operatorname{dim} K$.

The "Only if" part: By the criterion of symmetry, we have $\operatorname{dim} K=$ $\operatorname{dim} K^{\vee}$, that is, $d=d\left(d-\operatorname{dim} V_{n-1}\right)$. Hence $\operatorname{dim} V_{n-1}=d-1$. For $0 \leq$ $i \leq n-1$, we have the required equality $d-\operatorname{dim} V_{n-1-i}=\operatorname{dim} K / V_{n-1-i}=$ $\operatorname{dim} V_{i}^{\vee}=\operatorname{dim} V_{i}$.

The "If" part: Note that $K / V_{n-1}$ is injective over $\kappa$. It follows that $\operatorname{dim} V_{n-1}$ $=d-1$ and thus $\operatorname{dim} V_{i}^{\vee}=\operatorname{dim} V_{i}$ for all $i$. It is easy to see that the canonical map $K \hookrightarrow K^{\vee}$ is an isomorphism by comparing the dimensions. The composition of the canonical maps $K \rightarrow K / V_{n-1-i} \rightarrow V_{i}^{\vee}$ factors 
also as $K \stackrel{\sim}{\rightarrow} K^{\vee} \rightarrow V_{i}^{\vee}$. The kernel of the composition has dimension $d-\operatorname{dim} V_{i}=\operatorname{dim} V_{n-1-i}$. Since $V_{n-1-i} V_{i} \subset V_{n-1}$, the kernel is $V_{n-1-i}$. Thus, we have $K / V_{n-1-i} \cong V_{i}^{\vee}$. By the criterion of symmetry, $S$ is of injective dimension 1, and hence Gorenstein.

Note that, if $\operatorname{dim}_{\kappa} K=1$, the ring $S$ reduces to $K\left[\left[X^{t_{1}}, \ldots, X^{t_{m}}\right]\right]$, where the g.c.d. of $t_{1}, \ldots, t_{m}$ is 1 . The corollary becomes Kunz's criterion [10] that the Noetherian ring $S$ is Gorenstein if and only if the semigroup generated by $t_{1}, \ldots, t_{m}$ is symmetric. An immediate application of this corollary gives the following examples.

EXAmPLE 4.7. (1) Let $\kappa \subset K$ be a finite field extension. The Noetherian ring $S=\kappa \oplus \kappa X \oplus \cdots \oplus \kappa X^{n-1} \oplus K[[X]] X^{n}$ is Gorenstein if and only if $\operatorname{dim}_{\kappa} K \leq 2$.

(2) Let $K=\kappa[\alpha]$ be a finite field extension of degree $n+1$ over $\kappa$ and let $R_{i}=\kappa+\kappa \alpha+\cdots+\kappa \alpha^{i}$. Then the Noetherian ring $S=R_{0} \oplus R_{1} X \oplus \cdots \oplus$ $R_{n-1} X^{n-1} \oplus K[[X]] X^{n}$ is Gorenstein.

For the non-Noetherian case (for instance when $R_{0}$ is not a field), we need something to replace the dimension of vector spaces, which is used in [1], [3] to characterize Gorenstein rings.

Definition 4.8. Let $A$ be any commutative ring and let $M$ and $N$ be $A$ modules. We say $N$ is self-dual with respect to $M$ over $A$ if $N \cong \operatorname{Hom}_{A}(N, M)$. A domain $R$ is with self-dual quotients if $Q=Q(R)$ is self-dual with respect to $Q / R$ over $R$.

LEMMA 4.9. Consider the ring $S$ as given in (2) where $K$ is a finite extension of $Q=Q(R)$. If $K$ is self-dual with respect to $K / R_{n-1}$, then the canonical map $K \rightarrow K^{\vee}=\operatorname{Hom}_{R}\left(K, K / R_{n-1}\right)$ is an isomorphism.

Proof. Note that the canonical map is $Q$-linear since both $K$ and $K^{\vee}$ are $K$-modules and hence $Q$-modules. If $K$ is self-dual with respect to $K / R_{n-1}$, then the canonical map $K \hookrightarrow K^{\vee}$ is also an isomorphism since $\operatorname{dim}_{Q(R)} K^{\vee}=$ $\operatorname{dim}_{Q(R)} K<\infty$.

Proposition 4.10. Let $R$ be a domain and not a field and let

$$
S=R \oplus R X \oplus \cdots \oplus R X^{n-1} \oplus Q[[X]] X^{n},
$$

where $Q=Q(R)$. Then $S$ is of injective dimension 1 if and only if $R$ is of injective dimension 1 and with self-dual quotients.

If, in addition, $(R, \mathfrak{m})$ is a local domain and $Q / R$ is an injective hull of $R / \mathfrak{m}$, then $S$ is also a local domain with maximal ideal $\mathfrak{n}=\mathfrak{m}+(S \cap Q[[X]] X)$ and $Q((X)) / S$ is an injective hull of $S / \mathfrak{n}$. 
Proof. The first part of the statement follows from Lemma 4.9 and the criterion of symmetry. To see the second part, let $a=X^{n} Q[[X]]$. Then $Q((X)) / S \cong \operatorname{Hom}_{R}^{\mathfrak{a}}(S, Q / R)$ by Corollary 4.4. Let $\mathfrak{b}=S \cap X Q[[X]]$. Then $\mathfrak{b}$ is adically equivalent to $\mathfrak{a}$. By Corollary $3.5, Q((X)) / S$ is an injective hull of $\operatorname{Hom}_{R}(S / \mathfrak{b}, R / \mathfrak{m}) \cong R / \mathfrak{m} \cong S / \mathfrak{l}$ as $S$-modules.

Among rings of injective dimension 1, there are plenty of domains with self-dual quotients. We start with the Noetherian case.

Proposition 4.11. Let $R$ be a local Gorenstein domain of dimension 1. Then $R$ is complete if and only if it is with self-dual quotients.

Proof. Under our assumption, $Q / R$ is an injective hull of the residue field of $R$ and the functor ${ }^{\vee}=\operatorname{Hom}_{R}(-, Q / R)$ is the Matlis dual. We have a commutative diagram

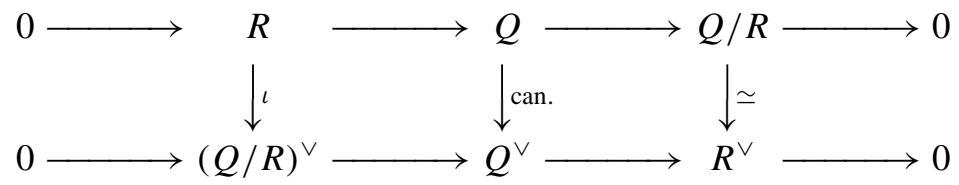

with exact rows, where the map $R \rightarrow(Q / R)^{\vee}$ is identified with the map from $R$ to its completion. From 5-lemma, $Q$ is self-dual with respect to $Q / R$ if and only if $\iota$ is an isomorphism if and only if $R$ is complete.

Self-duality of quotient fields is preserved under suitable base change.

Proposition 4.12 (Stability of Self-Duality). Let $R, S, K$ and a be as in (2) where $K=Q=Q(R)$. If $Q$ is self-dual with respect to an $R$-module $E$ (not necessarily injective), then $Q((X))$ is self-dual with respect to $\operatorname{Hom}_{R}^{\mathfrak{a}}(S, E)$ over $S$.

Proof. Let $\alpha: Q \rightarrow \operatorname{Hom}_{R}(Q, E)$ be an $R$-isomorphism. Write elements of $Q((X))$ as Laurent series of $X$. Let

$$
\Phi: Q((X)) \rightarrow \operatorname{Hom}_{S}\left(Q((X)), \operatorname{Hom}_{R}^{\mathfrak{a}}(S, E)\right)
$$

be the $S$-linear map given by $((\Phi(f))(g))(h)=(\alpha$ (the constant term of $f g h))(1)$, where $f, g \in Q((X))$ and $h \in S$. Clearly, $\Phi$ is one-to-one. To show that $\Phi$ is onto, suppose given an $S$-linear map $\varphi: Q((X)) \rightarrow \operatorname{Hom}_{R}^{\mathfrak{a}}(S, E)$. Observe first that

$$
(\varphi(g))(h)=(h \varphi(g))(1)=(\varphi(g h))(1),
$$

where $g \in Q((X))$ and $h \in S$. This implies that $\varphi$ is determined by the values $(\varphi(f))(1)$ for all $f \in Q((X))$. From linearity and the property that 
any element of $\operatorname{Hom}_{R}^{\mathfrak{a}}(S, E)$ is annihilated by sufficiently high powers of $X$, we observe furthermore that $\varphi$ is determined by the values $\left(\varphi\left(a X^{i}\right)\right)(1)$ for all $a \in Q$ and $i \in Z$ Z. In other words, $\varphi$ is determined by the $R$-linear maps $Q \rightarrow E$ given by $a \mapsto\left(\varphi\left(a X^{i}\right)\right)(1)$, where $i \in Z$. Let $a_{-i}$ be the preimage of the map $a \mapsto\left(\varphi\left(a X^{i}\right)\right)(1)$ under $\alpha$. Note that $a_{-i}=0$ for sufficiently large $i$. So we obtain an element $\sum a_{i} X^{i} \in Q((X))$. Note that $\alpha$ is $Q$-linear since $\operatorname{Hom}_{R}(Q, E)$ is a $Q$-module. For $a \in Q$,

$$
\left(\Phi\left(\sum a_{i} X^{i}\right)\right)\left(a X^{j}\right)(1)=\left(\alpha\left(a_{-j} a\right)\right)(1)=\left(\alpha\left(a_{-j}\right)\right)(a)=\left(\varphi\left(a X^{j}\right)\right)(1) .
$$

Hence $\Phi\left(\sum a_{i} X^{i}\right)=\varphi$.

Corollary 4.13. Let $S, R$ be as given in (4). If $R$ is of injective dimension 1 and with self-dual quotients, then so is $S$.

Proof. By Proposition 4.10, $Q / R$ is injective and $S$ is of injective dimension 1. By Proposition 4.12 and Corollary 4.4, the quotient field $Q((X))$ of $S$ is self-dual with respect to $Q((X)) / S$.

Thus, we may construct many domains of injective dimension 1 and with self-dual quotients. In particular, we may apply our results to the so-called $D+M$ construction.

ExAMPLe 4.14 (Iterated $D+M$ constructions). Let $\left(S_{1}, \mathfrak{m}_{1}\right)$ be a complete local Gorenstein domain of dimension 1 with the quotient field $K_{1}$. For $n \in Z_{+}$, let $\left(S_{n+1}, \mathfrak{m}_{n+1}\right)$ be the domain with the quotient field $K_{n+1}$ defined recursively by $S_{n+1}=S_{n} \oplus X_{n} K_{n}\left[\left[X_{n}\right]\right]$ where $X_{n}$ is an indeterminate over $K_{n}$. The ideal $\mathfrak{a}_{n+1}:=X_{n} K_{n}\left[\left[X_{n}\right]\right]$ is generated up to radical by $X_{n}$ and $\mathfrak{m}_{n+1}=\mathfrak{a}_{n+1}+\mathfrak{m}_{n}$. For each $n, S_{n}$ is a local domain of Krull dimension $n$.

We may prove by induction on $n$ using Proposition 4.11 and Corollary 4.13 that $S_{n}$ is of injective dimension 1 and with self-dual quotients. Furthermore, $K_{n} / S_{n}$ is an injective hull of the residue field of $S_{n}$.

Recall that a valuation ring is called almost maximal if and only if its quotient field modulo the ring is injective [4, Theorem 4.4, Chapter IX]. Since the $D+M$ construction also preserves valuation rings, we have a series of almost maximal valuation ring if $S_{1}$ is a complete DVR.

ExAmple 4.15. Let $R$ be a domain of injective dimension 1 and with selfdual quotients. From the discussions in this section, we see that $(R, R)$ and $(Q, 0)$ are both dual pairs with respect to $Q / R$. We may construct more domains of injective dimension 1 and with self-dual quotients using these dual pairs as long as $R_{n-1}=R$. For example, when $n \geq 4$ is even,

$$
S=R \oplus Q X^{n / 2} \oplus \cdots \oplus Q X^{n-2} \oplus R X^{n-1} \oplus Q[[X]] X^{n}
$$


is such a domain.

EXAMPLE 4.16. Using Lemma 4.9 and the criterion of symmetry, we can construct more domains of injective dimension 1 and with self-dual quotients, such as

$$
S=\kappa\left[\left[t^{2}\right]\right] \oplus \kappa((t)) X^{2} \oplus\left(\kappa\left[\left[t^{2}\right]\right]+\kappa\left(\left(t^{2}\right)\right) t\right) X^{3} \oplus \kappa((t))[[X]] X^{4}
$$

where $\kappa$ is a field and $t$ is an indeterminate over $\kappa$. We leave the details to the reader.

\section{REFERENCES}

1. Barucci, V., and Fröberg, R., Maximality properties for one-dimensional analytically irreducible local Gorenstein and Kunz rings, Math. Scand. 81 (1998), 149-160.

2. Brodmann, M. P., and Sharp, R. Y., Local Cohomology: an Algebraic Introduction with Geometric Applications, Cambridge Studies in Adv. Math. 60, Cambridge Univ. Press, Cambridge 1998.

3. Campillo, A., Delgado, F., and Kiyek, K., Gorenstein property and symmetry for onedimensional local Cohen-Macaulay rings, Manuscripta Math. 83 (1994), 405-423.

4. Fuchs, L., and Salce, L., Modules over Non-Noetherian Domains, Math. Surveys and Monographs 84, Amer. Math. Soc., Providence, RI 2001.

5. Hizem, S., and Benhissi, A., When is $A+X B[[X]]$ Noetherian? C. R. Math. Acad. Sci. Paris 340 (2005), 5-7.

6. Huang, I-C., Pseudofunctors on modules with zero dimensional support, Mem. Amer. Math. Soc. 114 (1995), no. 548.

7. Huang, I-C., A residue map and its applications to some one-dimensional rings, Proc. Amer. Math. Soc. 123 (1995), 2369-2372.

8. Huang, I-C., An explicit construction of residual complexes, J. Algebra 225 (2000), 698-739.

9. Kunz, E., Kähler Differentials, Vieweg, Braunschweig 1986.

10. Kunz, E., The value semigroup of a one-dimensional Gorenstein ring, Proc. Amer. Math. Soc. 25 (1970), 748-751.

11. Matsumura, H., Commutative Ring Theory, Cambridge Studies in Adv. Math. 8, Cambridge Univ. Press, Cambridge 1986.

12. Weibel, C. A., An Introduction to Homological Algebra, Cambridge Studies in Adv. Math. 38, Cambridge Univ. Press, Cambridge 1994.

DEPARTMENT OF MATHEMATICS

NATIONAL CHUNG CHENG UNIVERSITY

MINGHSIUNG, CHIAYI 621

TAIWAN, R.O.C

E-mail: stchang@math.ccu.edu.tw
INSTITUTE OF MATHEMATICS

ACADEMIA SINICA

6F, ASTRONOMY-MATHEMATICS BUILDING NO. 1 , SEC. 4 , ROOSEVELT ROAD TAIPEI 10617 TAIWAN, R.O.C.

E-mail: ichuang@math.sinica.edu.tw 